\title{
Is cardiovascular disease a risk factor in the development of axonal polyneuropathy?
}

\author{
L L Teunissen, H Franssen, J H J Wokke, Y van der Graaf, W H J P Linssen, J D Banga, \\ D M Laman, N C Notermans
}

See end of article for authors' affiliations

......................

Correspondence to: Dr Teunissen, University Medical Centre Utrecht,

Department of Neurology, G03.236, PO Box 85500

3508 GA Utrecht, The

Netherlands;

I.I.teunissen@neuro.azu.n

Received 28 June 2001

In revised form

27 November 2001

Accepted

13 December 2001
Objectives: To determine if cardiovascular disease may be a risk factor in the development of chronic idiopathic axonal polyneuropathy (CIAP).

Methods: In this incidence case-control study, the prevalence of cardiovascular disease and risk factors in 97 patients with CIAP (mean age 67.5 (SD 7.9) years) and the prevalence of neuropathic features in 97 patients with peripheral arterial disease (PAD) (mean age 67.1 (SD 7.3) years) were investigated. The results were compared with those for 96 age and sex matched controls without diagnosed PAD or polyneuropathy (mean age 67.5 (SD 9.1) years). In a randomly chosen subgroup of 23 patients with CIAP, 42 patients with $P A D$, and 48 controls, an electrodiagnostic investigation was performed.

Results: Patients with CIAP more often had manifest cardiovascular disease and cardiovascular risk factors than controls (stroke $18 \%$ v $6 \%$ of patients, odds ratio (OR) 3.2 (95\% confidence interval (CI) 1.8 to 5.9); heart disease $29 \%$ v $15 \%$, OR $2.4(95 \% \mathrm{Cl} 1.2$ to 4.9$)$; family history of cardiovascular disease $42 \%$ v $21 \%$, OR $2.8(95 \% \mathrm{Cl}(1.5$ to 5.2$)$; hypertension $56 \%$ v 39\%, OR $2.0195 \% \mathrm{Cl} 1.1$ to $3.6)$; hypercholesterolaemia $46 \%$ v $21 \%$, OR $3.3(95 \% \mathrm{Cl} 1.5$ to 7.3$)$; current smoking $38 \%$ v $23 \%$, OR $2.1(95 \% \mathrm{Cl} 1.1$ to 3.9)). The prevalence of cardiovascular disease and cardiovascular risk factors was lower than in patients with PAD. Patients with PAD more often had polyneuropathy than controls $(15 \%$ v 5\%, OR $3.3(95 \% \mathrm{Cl} 1.1$ to 10.0$))$. There was a trend towards lower nerve conduction velocities and lower amplitudes on electrodiagnostic investigation compared with controls.

Conclusion: This study shows that cardiovascular disease and CIAP often coexist, and therefore cardiovascular disease may be a cofactor in the development of CIAP.
$\mathrm{T}$ he prevalence of polyneuropathy is estimated to be between 2.4 and 8 per $1000 .{ }^{12}$ In $10-18 \%$ of patients, the cause of the polyneuropathy remains unknown, despite extensive evaluation. ${ }^{34}$ Most of these patients have a slowly progressive course. They share common clinical features: a relatively mild, predominantly sensory polyneuropathy with axonal degeneration, male predominance, and a mean age of onset of around 60 years. ${ }^{35-8}$ Although, by definition, patients with an unknown cause of polyneuropathy must form a heterogeneous group, considering the similarity in age and clinical features, these patients may share a common condition which causes or enhances the development of polyneuropathy. We have designated this condition chronic idiopathic axonal polyneuropathy (CIAP). ${ }^{6}$ Recently a similar group has been described as chronic cryptogenic sensory polyneuropathy. ${ }^{8}$

Animal studies have shown that acute and chronic ischaemia can lead to peripheral nerve lesions. ${ }^{9-12}$ In several clinical conditions, a relation between ischaemia or hypoxia and polyneuropathy is assumed. In patients with peripheral arterial disease (PAD) and chronic obstructive pulmonary disease, a high incidence of polyneuropathy is found. ${ }^{13-16}$ In diabetes mellitus, several studies indicate that ischaemia and metabolic factors together cause diabetic neuropathy. ${ }^{17} 18$ Abnormalities in the blood vessel wall, which are seen in diabetic polyneuropathy, ${ }^{19}$ chronic obstructive pulmonary disease, ${ }^{16}$ and $\mathrm{PAD},{ }^{14}$ were also found in patients with CIAP, being more prominent in those with manifest cardiovascular disease..$^{20}$ These observations together with the similarity in age and sex distribution of patients with CIAP and those with cardiovascular disease led us to hypothesise that cardiovascular disease may play a role in the development of CIAP.
To investigate whether there is a relation between cardiovascular disease and polyneuropathy, we performed an incidence case-control study to compare the prevalence of cardiovascular disease, cardiovascular risk factors, and neuropathic features in 97 patients with CIAP, 97 patients with PAD, and 96 age and sex matched controls.

\section{PATIENTS AND METHODS}

The study was designed as a case-control study with incidence cases. Patients were seen between 1995 and 1998. In total, 97 patients with CIAP, 97 patients with PAD, and 96 controls matched for age and sex were included (table 1). The same investigator (LLT) examined all patients and controls. A standardised history focused on complaints suggesting neuropathy, cardiovascular disease, and risk factors for cardiovascular disease. All patients and controls gave their informed consent, and the committee for human research of the University Medical Centre Utrecht approved the protocol.

\section{Polyneuropathy}

In this study, polyneuropathy was clinically defined as the presence of tingling, numbness, or loss of strength in combination with the following abnormalities at neurological examination: a symmetrical diminished sense of pain or touch, more prominent in the feet than in the hands, and two of the following abnormalities: loss of vibration sense in the

Abbreviations: $\mathrm{CIAP}$, chronic idiopathic axonal polyneuropathy; PAD, peripheral arterial disease; TIA, transient ischaemic attack; CMAP, compound muscle action potential; SNAP, sensory nerve action potential; $\mathrm{BMI}$, body mass index 
Table 1 Cardiovascular history and examination: comparison between patients with CIAP, patients with PAD, and controls

\begin{tabular}{|c|c|c|c|c|c|c|c|c|c|}
\hline & \multirow[b]{2}{*}{$\begin{array}{l}\text { CIAP } \\
(n=97)\end{array}$} & \multirow{2}{*}{$\begin{array}{l}\text { PAD } \\
(n=97)\end{array}$} & \multirow{2}{*}{$\begin{array}{l}\text { Controls } \\
(n=96)\end{array}$} & \multicolumn{2}{|c|}{ CIAP $v$ controls } & \multicolumn{2}{|c|}{ PAD $v$ controls } & \multicolumn{2}{|c|}{ CIAP $\vee P A D$} \\
\hline & & & & OR & $95 \% \mathrm{Cl}$ & OR & $95 \% \mathrm{Cl}$ & OR & $95 \% \mathrm{Cl}$ \\
\hline Men/women & $66 / 31$ & $61 / 36$ & $61 / 35$ & & & & & & \\
\hline Mean(SD) age (years) & $67.5(7.9)$ & $67.5(9.1)$ & $67.1(7.3)$ & & & & & & \\
\hline Fontaine I/II/III/IV & $12 / 9 / 0 / 0$ & $0 / 80 / 10 / 7$ & $8 / 1 / 0 / 0$ & & & & & & \\
\hline \multicolumn{10}{|l|}{ Cardiovascular risk factors } \\
\hline Family history positive & 42 & 37 & 21 & 2.8 & 1.5 to 5.2 & 2.2 & 1.2 to 4.3 & 1.2 & 0.7 to 2.2 \\
\hline Hypertension & 56 & 65 & 39 & 2.0 & 1.1 to 3.6 & 3.0 & 1.6 to 5.3 & 0.7 & 0.4 to 1.2 \\
\hline Hypercholesterolaemia & 46 & 60 & 21 & 3.3 & 1.5 to 7.3 & 5.7 & 2.6 to 12.4 & 0.6 & 0.3 to 1.2 \\
\hline Ever smoked & 68 & 92 & 63 & 1.1 & 0.8 to 1.4 & 10 & 3.6 to 25 & 0.1 & 0.04 to 0.3 \\
\hline Current smoking & 38 & 72 & 23 & 2.1 & 1.1 to 3.9 & 8.7 & 4.5 to 16.7 & 0.2 & 0.1 to 0.4 \\
\hline Alcohol use & 70 & 65 & 82 & 0.5 & 0.2 to 0.9 & 0.4 & 0.2 to 0.7 & 1.3 & 0.7 to 2.3 \\
\hline $\mathrm{BMI}>0.25$ & 44 & 45 & 39 & 1.1 & 0.8 to 1.5 & 1.3 & 0.7 to 2.3 & 0.9 & 0.5 to 1.6 \\
\hline \multicolumn{10}{|l|}{ Cardiovascular disease } \\
\hline Stroke & 17 & 18 & 6 & 3.2 & 1.8 to 5.9 & 3.4 & 1.3 to 9.0 & 0.9 & 0.4 to 1.9 \\
\hline Cardiac disease & 28 & 44 & 15 & 2.4 & 1.2 to 4.9 & 4.5 & 2.2 to 9.0 & 0.5 & 0.3 to 1.0 \\
\hline Intermittent claudication & 13 & 92 & 1 & & & & & & \\
\hline$A B \mid$ rest $<0.9$ & 10 & 100 & 1 & 10.8 & 1.4 to 86 & & & & \\
\hline $\mathrm{ABI}$ after exercise $<0.9$ & 15 & 100 & 8 & 2.3 & 0.9 to 5.7 & & & & \\
\hline Stroke and cardiac disease & 41 & 54 & 19 & 3.0 & 1.6 to 5.8 & 5.0 & 2.6 to 9.9 & 0.6 & 0.3 to 1.1 \\
\hline
\end{tabular}

Percentages of patients and controls with manifest cardiovascular disease and risk factors are presented. Statistically significant differences are presented in italics.

CIAP, chronic idiopathic axonal polyneuropathy; PAD, peripheral arterial disease; OR, odds ratio; $\mathrm{Cl}$, confidence interval; Fontaine stage I: subclinical $P A D$; stage II: pain after exercise, stage III: pain in rest, stage IV: ulceration; BMI, body mass index; $A B I$, ankle brachial index.

big toe, absent ankle jerks, and decreased muscle strength more prominent in the feet than in the hands. This definition was based on descriptions by us and others of a symmetrical polyneuropathy, more prominent in the distal part of the legs. ${ }^{821} 22$

\section{Chronic idiopathic axonal polyneuropathy (CIAP)}

Patients were referred to the outpatient clinics of the neurology departments of the University Medical Centre, Utrecht (78 patients) and St Lucas Andreas Hospital, Amsterdam (19 patients). CIAP was diagnosed if patients had a slowly progressive distal symmetric chronic polyneuropathy that at least fulfilled the above definition. No cause for the polyneuropathy was found after extensive clinical and laboratory evaluation including a search for monoclonal proteins, metabolic, endocrine, malignant, or autoimmune diseases, exposure to toxic agents, or hereditary causes. ${ }^{6}$ In all 97 patients with CIAP, the results of the electrodiagnostic investigation were consistent with an axonal polyneuropathy. ${ }^{6}$ No evidence of demyelination was found according to our criteria, ${ }^{23}$ which are modified from those of the American Academy of Neurology. ${ }^{24}$ Electrodiagnostic investigation was performed in all patients to confirm the presence of an axonal polyneuropathy. ${ }^{6}$ In a subset of patients, a sural nerve biopsy was performed to exclude vasculitis and amyloidosis. The results of these biopsies were presented in an earlier paper. ${ }^{20}$

\section{Peripheral arterial disease (PAD)}

Patients with PAD, visiting the outpatient vascular clinics of the University Medical Centre, Utrecht (57 patients) and St Lucas Andreas Hospital, Amsterdam (40 patients) were studied. All patients had enduring lifestyle limiting complaints due to PAD. All patients with PAD had an ankle-brachial index (ABI) $<0.90$ and at least Fontaine stage II (pain after exercise). Medical charts of all patients were reviewed and an extensive history was taken. Patients with a disease that could cause polyneuropathy-for example, diabetes mellitus, thyroid disease, or alcohol abuse-were not included. Diabetes had to be excluded in all patients with PAD before they were entered in the study. We chose the patients with PAD in such a way that they matched for age and sex the patients with CIAP.

\section{Controls}

Because our patients formed a selected group who were visiting specialised outpatient clinics for neuromuscular diseases of a university hospital and general hospital, we did not take random controls, but chose as controls partners or acquaintances of our patients. Controls who had a disease that could cause polyneuropathy (see above) or who were diagnosed as having PAD were excluded. No other restrictions to inclusion were applied, meaning that controls could have undiagnosed polyneuropathy or PAD. We chose controls in such a way that they matched for age and sex the patients with CIAP.

\section{Cardiovascular evaluation}

Clinically manifest cardiovascular disease was defined as ischaemic heart disease-that is, ischaemic chest pain or myocardial infarction-or ischaemic cerebral disease-that is, transient ischaemic attack (TIA) or cerebral infarction. Risk factors for cardiovascular disease were defined as hypercholesterolaemia (serum cholesterol level $>6.5 \mathrm{mmol} / \mathrm{l}$ or the use of cholesterol lowering agents), a positive family history (a first degree relative with cardiovascular disease at age $<65$ years), hypertension (use of antihypertensive drugs or a mean blood pressure over 95 diastolic and/or 160 systolic at two measurements), current daily smoking, and consumption of more than four glasses of alcohol a day. The ankle-brachial index was measured and used as an indicator of PAD. The ankle-brachial index is the ratio between the systolic blood pressure in the ankle and in the arm. An ankle-brachial index of over 1.00 is considered normal, and one below 0.90 at rest or after exercise was considered abnormal. ${ }^{25}{ }^{26}$ Intermittent claudication was assessed according to the Rose criteria, ${ }^{27}$ and severity defined as follows: Fontaine stage I, subclinical PAD; II, pain after exercise; III, pain at rest; IV, ulceration.

\section{Neurological evaluation}

The neurological examination was performed according to a standardised protocol ${ }^{6}$ : muscle strength, touch, pinprick, vibration, and joint position sense were measured in both arms and legs. The presence of atrophy of the intrinsic hand muscles and lower leg muscles was noted. A Romberg test was performed to measure stability. Ataxia was quantified by the tapping test for the dominant arm and leg as follows. A device 
consisting of two push buttons for the hands or two pedals for the feet was placed at a fixed distance of $35 \mathrm{~cm}$ and connected to an automatic counter. ${ }^{28}$ The patient had to alternately push buttons or pedals as fast as possible, and the number of hits in 15 seconds was counted. The vibration perception threshold was measured on the dominant metacarpal 2 using a Vibrameter type 3 (Somedic AB, Stockholm, Sweden). ${ }^{29}$ The modified Rankin scale was used to score disability. ${ }^{30}$

\section{Electrodiagnostic investigation}

In addition to the electrodiagnostic investigation performed in all 97 patients with CIAP, a randomly chosen subgroup of 23 patients with CIAP, 42 patients with PAD, and 48 controls underwent an electrodiagnostic investigation according to a standardised protocol. ${ }^{31}$ The limbs were warmed in water for 30 minutes before the investigation; thereafter skin temperature was maintained at $37^{\circ} \mathrm{C} .{ }^{32}$ Nerve conduction studies were performed using surface electrodes by standard techniques. ${ }^{33}$ Motor nerve conduction was investigated up to the axilla in the median nerve (recording: abductor pollicis brevis muscle) and up to the popliteal fossa in the tibial nerve (recording: abductor hallucis brevis muscle); the distance between the distal stimulation point and the active recording electrode was $7 \mathrm{~cm}$ and $10 \mathrm{~cm}$ respectively. Antidromic sensory nerve conduction was investigated in the median nerve (recording with ring electrodes from the second digit) and in the sural nerve. F waves were elicited by 20 stimuli at the wrist or ankle. Concentric needle electromyography was performed in the tibialis anterior muscle. The following were scored: distal motor latency; motor conduction velocities in the lower arm, upper arm, and lower leg segments; amplitude, duration, and area of the negative part of the compound muscle action potential (CMAP); reduction of CMAP amplitude and area, and increase in CMAP duration after distal compared with proximal stimulation of each segment; minimal F-M latency; sensory conduction velocities and amplitude of the negative part of the sensory nerve action potential (SNAP) after distal stimulation; presence of fibrillations or positive sharp waves on concentric needle electromyography.

\section{Statistical analysis}

Odds ratios (OR) and 95\% confidence intervals (95\% CI) were calculated to assess differences between groups. ORs were considered significant if the $95 \%$ CI did not include 1.0. The means of the electrodiagnostic variables, vibration threshold, and tapping test results were compared with the MannWhitney $U$ test for non-parametric values. Results were considered significant if $\mathrm{p}<0.05$.

\section{RESULTS \\ Cardiovascular history and examination}

Patients with CIAP compared with controls

Overall, $17 \%$ of patients with CIAP had suffered from a stroke (9\% TIA, $8 \%$ cerebral infarction) and $28 \%$ from ischaemic heart disease ( $13 \%$ ischaemic chest pain, $15 \%$ myocardial infarction), compared with $6 \%$ with strokes (3\% TIA, 3\% cerebral infarction) and $15 \%$ with ischaemic heart disease ( $8 \%$ ischaemic chest pain, 7\% myocardial infarction) in controls. All investigated variables of cardiovascular disease and cardiovascular risk factors were significantly more common in patients with CIAP than in controls, except for an abnormal ankle-brachial index after exercise, body mass index (BMI), and whether the subject had ever smoked, which did not show a significant difference, and use of alcohol, which was significantly less common in patients with CIAP (table 1). None of the patients with CIAP or controls admitted consuming more than four glasses of alcohol a day.
Patients with PAD compared with controls

Overall, $18 \%$ of patients with PAD had suffered from a stroke ( $11 \%$ TIA, $7 \%$ cerebral infarction) and $44 \%$ from ischaemic heart disease (22\% ischaemic chest pain, $22 \%$ myocardial infarction). All investigated variables of cardiovascular disease and cardiovascular risk factors were significantly more common in patients with PAD than in controls, except for BMI, which did not show a significant difference, and use of alcohol, which was significantly less common in patients with PAD (table 1). None of the patients with PAD admitted consuming more than four glasses of alcohol a day.

Patients with CIAP compared with patients with PAD

Although all investigated variables except family history of cardiovascular diseases and alcohol use were more common in patients with PAD than in those with CIAP, only smoking (current and ever) reached a significant level (table 1).

\section{Neurological history and examination}

\section{Patients with CIAP compared with controls}

All investigated neuropathic symptoms and signs were significantly more common in patients with CIAP than in controls (table 2). Although neuropathic features were often present in controls, only 5\% fulfilled the defined criteria of polyneuropathy.

\section{Patients with PAD compared with controls}

All investigated neuropathic symptoms and signs were significantly more common in patients with PAD than in controls, except for complaints of muscle weakness, atrophy of the intrinsic muscles of the hand, absent biceps, triceps, knee, and ankle jerk, abnormal pin prick and touch sense (table 2). Fifteen percent of patients with PAD fulfilled the defined criteria of polyneuropathy.

Patients with PAD compared with patients with CIAP All investigated neuropathic symptoms and signs were significantly more common in patients with CIAP than in patients with PAD, except for complaints of pain, Rankin score, and the tapping test of the arm (table 2). By definition, all patients with CIAP fulfilled the criteria of polyneuropathy.

\section{Electrodiagnostic investigation}

Sex and age distribution in the subgroup of patients who underwent the standardised electrodiagnostic investigation were comparable to the total group (CIAP: $78 \%$ men, mean age 65.9 (SD 6.7) years; PAD: 71\% men, 67.0 (SD 10.5) years; controls: $65 \%$ men, 67.5 (SD 6.6) years). The number of patients with severe PAD (Fontaine stage III or IV) was smaller than the total group of patients with PAD (CIAP: 17\% Fontaine stage I; PAD: $88 \%$ stage II, $7 \%$ stage III, $5 \%$ stage IV; controls: $2 \%$ stage I).

Patients with CIAP compared with controls

All variables were significantly worse in patients with CIAP than in controls, except for the sural nerve conduction velocity (table 3).

Patients with PAD compared with controls

There was a trend towards worse values for all variables in patients with PAD, in comparison with controls. However, only the median nerve motor conduction velocity in the lower arm and the tibial nerve motor conduction velocity, minimal F-M latency of the median nerve, and SNAP amplitude of the sural nerve were significantly worse in patients with PAD than in controls (table 3 ).

Patients with CIAP compared with patients with PAD The variables in patients with CIAP were all significantly worse than in patients with PAD, except for the median nerve CMAP amplitude after stimulation at the wrist and the sural nerve conduction velocity. None of the patients or controls had evidence of demyelination according to our criteria. 
Table 2 Neurological history and examination: comparison between patients with CIAP, patients with PAD, and controls

\begin{tabular}{|c|c|c|c|c|c|c|c|c|c|}
\hline & \multirow{2}{*}{$\begin{array}{l}\text { CIAP } \\
(n=97)\end{array}$} & \multirow{2}{*}{$\begin{array}{l}\text { PAD } \\
(n=97)\end{array}$} & \multirow{2}{*}{$\begin{array}{l}\text { Controls } \\
(n=96)\end{array}$} & \multicolumn{2}{|c|}{ CIAP $v$ controls } & \multicolumn{2}{|c|}{ PAD $v$ controls } & \multicolumn{2}{|c|}{ CIAP $\vee P A D$} \\
\hline & & & & OR & $\mathrm{Cl}$ & OR & $\mathrm{Cl}$ & OR & $\mathrm{Cl}$ \\
\hline \multicolumn{10}{|l|}{ Symptoms (\%) } \\
\hline Tingling & 57 & 33 & 17 & 6.5 & 3.3 to 12.8 & 2.5 & 1.2 to 4.9 & 2.7 & 1.5 to 4.8 \\
\hline Numbness & 80 & 32 & 16 & 22.2 & 10.5 to 46.7 & 2.5 & 1.2 to 5.1 & 9.0 & 4.5 to 16.7 \\
\hline Weakness & 72 & 11 & 11 & 20.0 & 9.3 to 43.2 & 1.0 & 0.4 to 2.4 & 20.4 & 9.4 to 43.5 \\
\hline Muscle cramps & 64 & 49 & 31 & 3.9 & 2.1 to 7.1 & 2.1 & 1.1 to 3.7 & 1.9 & 1.1 to 3.3 \\
\hline \multicolumn{10}{|l|}{ Disability (\%) } \\
\hline Walking distance $<1000 \mathrm{~m}$ & 21 & 62 & 1 & 24.7 & 3.2 to 188 & 154 & 20.6 to 1153 & 0.2 & 0.1 to 0.3 \\
\hline Use of walking aids & 39 & 14 & 4 & 14.8 & 5.0 to 43.7 & 3.9 & 1.2 to 12.3 & 3.8 & 1.9 to 7.7 \\
\hline Rankin $0 / 1 / 2 / 3$ & $0 / 31 / 64 / 5$ & $4 / 31 / 60 / 5$ & $69 / 27 / 4 / 0$ & & & & & & \\
\hline Rankin $>1$ & 69 & 65 & 4 & 7.2 & 4.1 to 12.4 & 42.6 & 14.4 to 127 & 1.1 & 0.6 to 2.0 \\
\hline \multicolumn{10}{|l|}{ Signs $(\%)$} \\
\hline Atrophy hands & 29 & 7 & 7 & 5.2 & 2.1 to 12.5 & 1.0 & 0.3 to 3.0 & 5.2 & 2.2 to 12.6 \\
\hline Atrophy lower legs & 54 & 33 & 11 & 8.9 & 4.2 to 18.8 & 3.8 & 1.8 to 8.2 & 2.3 & 1.3 to 4.2 \\
\hline Weakness extension feet & 70 & 19 & 1 & 223 & 29.6 to 1675 & 21.9 & 2.9 to 168 & 10.2 & 5.2 to 19.9 \\
\hline Ankle jerk absent & 87 & 49 & 35 & 11.8 & 5.7 to 24.2 & 1.7 & 1.0 to 3.1 & 6.9 & 3.4 to 13.9 \\
\hline Pin prick sense abnormal & 79 & 38 & 18 & 17.9 & 8.7 to 36.7 & 1.4 & 0.8 to 2.4 & 6.1 & 3.2 to 11.7 \\
\hline Touch sense abnormal & 80 & 36 & 19 & 17.9 & 8.7 to 36.4 & 1.3 & 0.8 to 2.1 & 7.2 & 3.7 to 13.7 \\
\hline Vibration sense hallux absent & 94 & 41 & 26 & 18.4 & 8.7 to 38.5 & 2.0 & 1.1 to 3.7 & 21.2 & 8.5 to 53.2 \\
\hline Neuropathy (\%) & 100 & 15 & 5 & & & 3.3 & 1.1 to 10 & & \\
\hline \multicolumn{10}{|l|}{ Tests (mean (SD)) } \\
\hline Vibration threshold & $2.0(2.0)$ & $1.4(1.2)$ & $0.9(0.8)$ & $p<0.001$ & & $p<0.01$ & & $p<0.05$ & \\
\hline Tapping arm & $29(8)$ & $31(5)$ & $35(7)$ & $p<0.001$ & & $p<0.01$ & & NS & \\
\hline Tapping leg & 29 (11) & $34(8)$ & $39(8)$ & $p<0.001$ & & $p<0.01$ & & $p<0.05$ & \\
\hline
\end{tabular}

Percentages of patients and controls with neuropathic signs and symptoms are presented. Significant differences are presented in italics. $\mathrm{CIAP}$, Chronic idiopathic axonal polyneuropathy; PAD, peripheral arterial disease; OR, odds ratio; $\mathrm{Cl}$, confidence interval.

Table 3 Electrodiagnosis: comparison between patients with CIAP, patients with PAD, and controls

\begin{tabular}{|c|c|c|c|c|c|c|}
\hline & $\begin{array}{l}\text { CIAP } \\
(n=23)\end{array}$ & $\begin{array}{l}\text { PAD } \\
(n=42)\end{array}$ & $\begin{array}{l}\text { Controls } \\
(n=48)\end{array}$ & $\begin{array}{l}\text { CIAP v } \\
\text { controls }\end{array}$ & $\begin{array}{l}\text { PAD v } \\
\text { controls }\end{array}$ & $\begin{array}{l}\text { CIAP v } \\
\text { PAD }\end{array}$ \\
\hline \multicolumn{7}{|l|}{ Median nerve, motor } \\
\hline $\mathrm{DML}(\mathrm{ms})$ & $4.4(0.7)$ & $3.8(0.5)$ & $3.7(0.4)$ & $p<0.001$ & NS & $p<0.01$ \\
\hline MCV lower arm $(\mathrm{m} / \mathrm{s})$ & $50(4.6)$ & $55(4.0)$ & $57(4.2)$ & $p<0.001$ & $p<0.01$ & $p<0.00$ \\
\hline MCV upper arm $(\mathrm{m} / \mathrm{s})$ & 55 (8.9) & 60 (6.7) & $62(5.1)$ & $p<0.001$ & NS & $p<0.05$ \\
\hline CMAP amplitude wrist (mV) & $8.3(2.8)$ & $9.7(4.1)$ & $10.7(2.9)$ & $p<0.01$ & NS & NS \\
\hline Minimal F-M latency (ms) & $27.1(2.8)$ & $25.0(2.1)$ & $23.7(2.0)$ & $p<0.001$ & $p<0.01$ & $p<0.01$ \\
\hline \multicolumn{7}{|l|}{ Median nerve, sensory } \\
\hline $\mathrm{SCV}$ hand $(\mathrm{m} / \mathrm{s})$ & $46(5.4)$ & $52(6.1)$ & $54(5.0)$ & $p<0.001$ & NS & $p<0.01$ \\
\hline SCV lower arm $(\mathrm{m} / \mathrm{s})$ & $49(6.5)$ & $57(4.1)$ & $58(4.1)$ & $p<0.001$ & NS & $p<0.00$ \\
\hline SNAP amplitude wrist (uV) & $7.9(6.8)$ & $13.8(7.9)$ & $16.1(7.7)$ & $p<0.001$ & NS & $p<0.01$ \\
\hline \multicolumn{7}{|l|}{ Tibial nerve } \\
\hline $\mathrm{DML}(\mathrm{ms})$ & $4.8(0.8)$ & $4.3(0.9)$ & $4.0(0.4)$ & $p<0.001$ & NS & $p<0.01$ \\
\hline$M C V(\mathrm{~m} / \mathrm{s})$ & $37(4.7)$ & $43(5.3)$ & $45(4.0)$ & $p<0.001$ & $p<0.05$ & $p<0.001$ \\
\hline CMAP amplitude ankle $(\mathrm{mV})$ & $1.8(2.3)$ & $7.0(5.1)$ & $8.0(3.5)$ & $p<0.001$ & NS & $p<0.001$ \\
\hline \multirow{2}{*}{ Minimal F-M latency (ms) } & $58.7(7.2)$ & $49.6(6.3)$ & $48.6(5.1)$ & $p<0.001$ & NS & $p<0.00$ \\
\hline & $N R=22 \%$ & $N R=0 \%$ & $N R=0 \%$ & & & \\
\hline \multicolumn{7}{|l|}{ Sural nerve } \\
\hline $\mathrm{SCV}(\mathrm{m} / \mathrm{s})$ & $46(2.9)$ & $47(5.3)$ & $49(5.1)$ & NS & NS & NS \\
\hline SNAP amplitude (uV) & $3.8(1.8)$ & $9.3(6.6)$ & $11.8(6.0)$ & $p<0.001$ & $p<0.05$ & $p<0.01$ \\
\hline & $N R=52 \%$ & $N R=7 \%$ & $N R=2 \%$ & & & \\
\hline \multicolumn{7}{|c|}{ Electromyography anterior tibial muscle } \\
\hline Spontaneous activity & 65 & 12 & 2 & $p<0.001$ & NS & $p<0.001$ \\
\hline
\end{tabular}

\section{DISCUSSION}

In this study, cardiovascular disease and cardiovascular risk factors were significantly more common in patients with CIAP, and neuropathic features were significantly more common in patients with PAD, compared with non-disease controls. Because patients were referred because of polyneuropathy of unknown cause and not because of other conditions, we presumed that cardiovascular disease did not form a selection bias. Because most patients with CIAP had mild complaints (Rankin 1 or 2 ), we thought it unlikely that the number of cardiovascular risk factors was increased by inactivity or increased body weight caused by general disease or pain. There were no differences in BMI between the groups. The numbers of patients with CIAP and controls who had ever smoked were similar, meaning that this did not form a selection bias. Because our patients formed a selected population, we decided to choose partners and acquaintances as controls instead of random controls.

In experimental models, chronic ischaemia can lead to axonal degeneration. ${ }^{10-12}$ Clinical and morphological signs of 
neuropathy have been found in patients with $\mathrm{PAD}$, related toseverity of ischaemia. ${ }^{13-15}$ These studies emphasise neurophysiological and morphological findings, and the clinical studies lack comparisons with normal controls and polyneuropathy controls. A high prevalence of polyneuropathy has also been found in patients with chronic obstructive pulmonary disease, possibly related to hypoxia. ${ }^{16}$ In diabetes mellitus, there is evidence that ischaemia, along with metabolic factors, plays a role in the development of diabetic neuropathy. ${ }^{17}{ }^{18}$ PAD aggravates diabetic neuropathy, ${ }^{34} 35$ and the incidence of diabetic neuropathy is higher in the presence of cardiovascular risk factors. ${ }^{36}{ }^{37}$ Reduction in the endoneurial blood flow, ${ }^{10}{ }^{11}$ hypotension, ${ }^{38}$ hypoxia of the blood, ${ }^{12}{ }^{16}$ and abnormalities in the blood vessel wall ${ }^{19}$ can all lead to insufficient oxygenation of peripheral nerves. Abnormalities of epineurial and endoneurial vessel walls are seen in several conditions, of which diabetes, ${ }^{19}$ chronic obstructive pulmonary disease, ${ }^{16}$ and PAD $^{13}{ }^{14}$ are ones in which ischaemia and hypoxia play a role. These abnormalities are thought to hamper oxygen transport and change the blood/nerve barrier. Abnormalities of the endoneurial vessel wall are also seen in sural nerve biopsy specimens of patients with CIAP, and were more prominent in patients with CIAP who had (subclinical) PAD. ${ }^{20}$

In our study, patients with PAD showed abnormalities suggestive of axonal degeneration on electrodiagnostic examination, but less commonly than in patients with CIAP. In earlier studies, slight slowing of conduction velocity and decreased SNAP and CMAP amplitudes were found in patients with PAD. ${ }^{15}{ }^{39}$ In our study, the differences between patients and controls were smaller than in earlier studies, probably because in our study patients had less severe PAD. Furthermore, in our protocol, $^{32}$ limbs were warmed in water, which may have improved the conduction velocity. Neuropathic features were also detected in the control group, and five cases even fulfilled our criteria for polyneuropathy. A higher prevalence of polyneuropathy has been reported in an elderly general population, ${ }^{1}$ and the loss of ankle reflexes and diminished vibration sense at the hallux increase with age. ${ }^{40}$ Moreover, we did not incorporate severity or duration of complaints into our criteria.

We conclude that cardiovascular disease may be a risk factor for the development of CIAP. The common concurrence of CIAP with cardiovascular disease and the presence of neuropathic features in patients with PAD suggest that our results are more than a confounder. However, the less common and less severe presence of neuropathic features in patients with PAD suggests that, as well as vascular disease, other unknown factors play an important role in the development of CIAP, and that CIAP has a multifactorial origin, with factors such as genetic disposition, aging, and lifestyle influencing the development of axonal degeneration.

\section{ACKNOWLEDGEMENTS}

We thank Dr A Voorwinde for referring patients with peripheral arterial disease and $\operatorname{Dr} G$ Wieneke for help with analysis of the electrophysiological data.

\section{Authors' affiliations}

L L Teunissen, J H J Wokke, N C Notermans, Department of Neurology, University Medical Centre, Utrecht, The Netherlands H Franssen, Clinical Neurophysiology, University Medical Centre, Utrecht

Y van der Graaf, Julius Centre for Patient Oriented Research, University Medical Centre, Utrecht

W H J P Linssen, Department of Neurology, St Lucas Andreas Hospital, Amsterdam, The Netherlands

J D Banga, Department of Internal Medicine, University Medical Centre, Utrecht

D M Laman, Department of Clinical Neurophysiology, St Lucas Andreas Hospital , Amsterdam

\section{REFERENCES}

1 IGPSG. Chronic symmetric symptomatic polyneuropathy in the elderly: a field screening investigation in two Italian regions. I. Prevalence and general characteristics of the sample. Italian General Practitioner Study (IGPSG). Neurology 1995;45:1832-6.

2 Martyn CN, Hughes RA. Epidemiology of peripheral neuropathy. J Neurol Neurosurg Psychiatry 1997;62:310-18.

3 McLeod JG, Tuck RR, Pollard JD, et al. Chronic polyneuropathy of undetermined cause. I Neurol Neurosurg Psychiatry 1984:47:530-5.

4 Grahmann F, Winterholler M, Neundorfer B. Cryptogenetic polyneuropathies: an out-patient follow-up study. Acta Neurol Scand 1991;84:221-5.

5 Dyck PJ, Oviatt KF, Lambert EH. Intensive evaluation of referred unclassified neuropathies yields improved diagnosis. Ann Neurol $1981 ; 10: 222-6$

6 Notermans NC, Wokke JH, Franssen $\mathrm{H}$, et al. Chronic idiopathic polyneuropathy presenting in middle or old age: a clinical and electrophysiological study of 75 patients. J Neurol Neurosurg Psychiatry 1993:56:1066-71.

7 Notermans NC, Wokke JH, van der Graaf Y, et al. Chronic idiopathic axonal polyneuropathy: a five year follow up. J Neurol Neurosurg Psychiatry 1994;57:1525-7

8 Wolfe GI, Baker NS, Amato AA, et al. Chronic cryptogenic sensory polyneuropathy, clinical and laboratory characteristics. Arch Neurol 1999:56:540-7.

9 Korthals JK, Wisniewski HM. Peripheral nerve ischemia. Part 1 Experimental model. J Neurol Sci 1975;24:65-76

10 Sladky JT, Tschoepe RL, Greenberg JH, et al. Peripheral neuropathy after chronic endoneurial ischemia. Ann Neurol 1991;29:272-8.

11 Sasaki H, Kihara M, Zollman PJ, et al. Chronic constriction model of rat sciatic nerve: nerve blood flow, morphologic and biochemical alterations. Acta Neuropathol Berl 1997;93:62-70.

12 Low PA, Schmelzer JD, Ward KK, et al. Experimental chronic hypoxic neuropathy: relevance to diabetic neuropathy. Am J Physiol 1986;250:E94-9

13 Eames RA, Lange LS. Clinical and pathological study of ischaemic neuropathy. J Neurol Neurosurg Psychiatry 1967;30:215-26.

14 Nukada H, van Rij AM, Packer SG, et al. Pathology of acute and chronic ischaemic neuropathy in atherosclerotic peripheral vascular disease. Brain 1996;119:1449-60.

15 Laghi Pasini F, Pastorelli M, Beermann U, et al. Peripheral neuropathy associated with ischemic vascular disease of the lower limbs. Angiology 1996;47:569-77

16 Nowak D, Bruch M, Arnaud F, et al. Peripheral neuropathies in patients with chronic obstructive pulmonary disease: a multicenter prevalence study. Lung 1990;168:43-51.

17 Tesfaye S, Malik R, Ward JD. Vascular factors in diabetic neuropathy. Diabetologia 1994;37:847-54.

18 Stevens MJ, Feldman EL, Greene DA. The aetiology of diabetic neuropathy: the combined roles of metabolic and vascular defects. Diabet Med 1995; 12:566-79.

19 Giannini C, Dyck PJ. Basement membrane reduplication and pericyte degeneration precede development of diabetic polyneuropathy and are associated with its severity. Ann Neurol 1995;37:498-504.

20 Teunissen LL, Notermans NC, Jansen GH, et al. Thickness of endoneurial vessel basement membrane in chronic idiopathic axonal polyneuropathy. Acta Neuropathol 2000;100:445-50.

21 Notermans NC, Wokke JHJ, Jennekens FGI. Clinical work-up of the patient with a polyneuropathy. In: de Jong JMBV, ed. Handbook of Clinical neurology. Hereditary neuropathies and spinocerebellar atrophies. Amsterdam: Elseviers Science Publishers, 1991:32-70.

22 Thomas PK, Ochoa J. Clinical features and differential diagnosis. In: Dyck PJ, Thomas PK, Griffin JW, eds. Peripheral neuropathy. Philadelphia: Saunders, 1993:749-74.

23 Van den Berg LH, Franssen H, Wokke JH. The long-term effect of intravenous immunoglobulin treatment in multifocal motor neuropathy. Brain 1998;121:421-8.

24 Ad Hoc Subcommittee of the American Academy of Neurology AIDS Task Force: Research criteria for diagnosis of chronic inflammatory demyelinating polyneuropathy. Neurology 1991;41:617-18

25 Stoffers H, Kester A, Kaiser V, et al. The diagnostic value of the measurement of the ankle-brachial systolic pressure index in primary health care. J Clin Epidemiol 1996;49:1401-5.

26 Newman AB, Siscovick DS, Manolio TA, et al. Ankle-arm index as a marker of atherosclerosis in the cardiovascular health study. Circulation 1993:88:837-45.

27 Rose GA, Blackburn RJ, Gillum RF, et al. Cardiovascular survey methods. Geneve: World Health Organization, 1982;162-5.

28 Notermans NC, van Dijk GW, van der Graaf Y, et al. Measuring ataxia: quantification based on the standard neurological examination. $J$ Neurol Neurosurg Psychiatry 1994;57:22-6.

29 Goldberg JM, Lindblom W. Standardised method of determining vibratory perception thresholds for diagnosis and screening in neurological investigation. J Neurol Neurosurg Psychiatry 1979:42:793-803.

30 Van Swieten JC, Koudstaal PJ, Visser MC, et al. Interobserver agreement for assessment of handicap in stroke patients. Stroke 1988; 19:604-7.

31 Teunissen LL, Notermans NC, Franssen H, et al. Differences between hereditary motor and sensory neuropathy type 2 and chronic idiopathic axonal neuropathy. A clinical and electrophysiological study. Brain 1997; 120:955-62 
32 Franssen $\mathbf{H}$, Wieneke $\mathrm{GH}$. Nerve conduction and temperature: necessary warming time. Muscle Nerve 1994;17:336-44.

33 Kimura J. Electrodiagnosis in diseases of nerve and muscle: principles and practice. Philadelphia: FA Davis, 1989:103-28.

34 Ram Z, Sadeh M, Walden R, et al. Vascular insufficiency quantitatively aggravates diabetic neuropathy. Arch Neurol 1991;48:1239-42.

35 Veves A, Donaghue VM, Sarnow MR, et al. The impact of reversal of hypoxia by revascularization on the peripheral nerve function of diabetic patients. Diabetologia 1996;39:344-8.

36 Harris M, Eastman R, Cowie C. Symptoms of sensory neuropathy in adults with NIDDM in the U.S. population. Diabetes Care 1993; 16:1446-52.
37 Forrest KY, Maser RE, Pambianco G, et al. Hypertension as a risk factor for diabetic neuropathy: a prospective study. Diabetes 1997;46:665-70.

38 Low PA, Tuck RR. Effects of changes of blood pressure, respiratory acidosis and hypoxia on blood flow in the sciatic nerve of the rat. $J$ Physiol (Lond) 1984;347:513-24.

39 Papapetropoulou V, Tsolakis J, Terzis S, et al. Neurophysiologic studies in peripheral arterial disease. J Clin Neurophysiol 1998;15:447-50.

40 Albert ML. Clinical neurology of aging. New York, Oxford: Oxford University Press, 1992.

\section{HISTORICAL NOTES \\ Cheyne-Stokes respiration}

$\mathrm{N}$

eurologists who care for emergencies often encounter CheyneStokes respiration. Abnormal breathing, in which periods of apnoea alternate with a series of gradually increasing depth and frequency, followed by a similar decrease is said to have been described by Hippocrates, although his description is not wholly convincing. ${ }^{1}$ It is often observed in grave cerebral illnesses. Dr J B Lyons ${ }^{2}$ has written informative details of the works of both Cheyne and Stokes.

In 1818, John Cheyne described ${ }^{4}$ a 60 year old man, who suffered from gout who he said was:

"of a sanguine temperament, circular chest, and full habit of body, for years had lived a very sedentary life, while he indulged habitually in the luxuries of the table."

He complained of palpitations and pain in the chest. He had fallen from a chair, but could not remember doing so. Cheyne found an "extremely irregular and unequal pulse" on examination, and the patient was confused and had a headache. After blood letting and the use of leeches, he improved.

"On the 10th of April he was found in bed, speechless, and hemiplegiac. ... The only peculiarity in the last period of his illness, which lasted eight or nine days, was in the state of the respiration. For several days, his breathing was irregular; it would cease for a quarter of a minute, then it would become perceptible, though very low, then by degrees it became heaving and quick, and then it would gradually cease again. This revolution in the state of his breathing occupied about a minute, during which there were about thirty acts of respiration"

Cheyne diagnosed apoplexy "that must have depended upon increased action of the vessels of the head". At post mortem examination was found a left cerebral infarct:

“. . . some fluid between it [the arachnoid] and the pia mater, and the vascularity of the latter increased, more particularly over the middle and posterior lobes of the cerebrum of the left side, where, in a large patch, it was thickened and of a deep red colour"

The heart was three times larger than normal, the left ventricle greatly enlarged, the right ventricle converted into a soft fatty substance-aorta steatomatous.

John Cheyne (1777-1836) studied medicine at Edinburgh, ${ }^{2}$ where Alexander Monro (1733-1817), who described the interventricular foramen, was one of his tutors. In 1809 he moved to Dublin where he was appointed physician to the Meath Hospital and professor of medicine at the College of Surgeons. He subsequently became Physician General in the Irish army in 1820, the highest medical ranking in Ireland. ${ }^{1}$ He was a founder of the Dublin Hospital reports, in which he described the foundation of Cheyne-Stokes respiration. He wrote books on croup, Essays on diseases of children, and An essay on hydrocephalus acutus, or dropsy in the brain. In his Cases of apoplexy and lethargy, he distinguished subarachnoid from intracerebral haemorrhage, probably the first physician to provide an illustration of subarachnoid haemorrhage.

Thirty six years later, in one of several distinguished texts, ${ }^{5}$ William Stokes described a disorder of the pattern of respiration, not caused by a lung condition, but by an enfeebled heart, due to fatty degeneration of this organ or other causes ${ }^{1}$ :

"The symptom in question was observed by Dr Cheyne, although he did not connect it with the special lesion of the heart. It consists in the occurrence of a series of inspirations, increasing to a maximum, and then declining in force and length, until a state of apparent apnoea is established. In this condition the patient may remain for such a length of time as to make his attendants believe that he is dead, when a low inspiration, followed by one more decided, marks the commencement of a new ascending and then descending series of inspirations. This symptom ... I have only seen during a few weeks previous to the death of the patient...."

He noted that a coexistence with disease of the aortic valve was common.

William Stokes (1804-1878) ${ }^{3}$ was born in 1804, the son of a physician, Whitley Stokes, MD (1763-1845), a distinguished and religious polymath, who became Regius Professor of Physic at Dublin University in 1830. Many of his family achieved prominence in academic society. He was taught privately by Rev John Walker in classics and mathematics, his father eschewing formal education. Stokes succeeded his father as Regius Professor of Medicine in Dublin in 1842. He received many honours, including those of the Universities of Oxford, Cambridge, and Edinburgh. By commendation of the English ambassador, he was decorated by the Emperor Wilhelm I with the Prussian order Pour le Mérite for contributions to medicine.

William Stokes, an original and outstanding physician, is also well known for his account of the Stokes-Adams syndrome.

J M S Pearce

304 Beverley Road, Anlaby, Hull HU10 7BG, UK; imspearce@freenet.co.uk

\section{References}

1 Major RH. Classic descriptions of disease. 3rd edn 6th printing Springfield: Thomas, 1965:548-53.

2 Lyons JB. John Cheyne's classic monographs. J Hist Neurosci 1995;4:27-35.

3 Lyons JB. A great Dublin medical family. Proceedings of the XXIII congress of the history of medicine, London, 2-9 September 1972. 1010-6.

4 Cheyne J. A case of apoplexy in which the fleshy part of the heart was converted into fat. Dublin Hospital Reports 1818;2:216-23.

5 Stokes W. The diseases of the heart and the aorta. Dublin: Hodges and Smith, 1854:323-4

6 Adams R. Cases of diseases of the heart. Dublin Hospital Reports $1827: 4: 396-453$ 